\title{
Association of range of accommodation with fully corrected refractive errors
}

\author{
Abstract \\ Background: To study whether the range of accommodation differs in myopes and \\ hypermetropes than normal population.
}

Purpose: To study the range of accommodation in myopes and hypermetropes in comparison to normal population in different age groups.

Materials and methods: A comparative cross-sectional study was conducted at MHL from January 2018 to October 2018. Total 99 patients were enrolled in this study who had refractive errors. All patients were included who had $10-55$ years age. Both genders were included. Both myopic and hypermetropic patients were included having fully corrected myopia and hypermetropia. Patients with any ocular pathologies were excluded. All patients who were included in this study had complete ocular and posterior chamber examination from ophthalmologist. There visual acuity was recorded using Snellen`s visual acuity chart and recorded in Snellen notation. Near vision was assessed on near vision chart. Then range of accommodation will be measured by RAF meter. All data entered and analyzed by SPSS 20 (Statically package for social scientist). Bar charts and Pie charts were used to describe qualitative data and range, mean, S.Dwere used to express quantitative data. For data normality Kolmogorov-Smirnov and Shapiro Wilktest used and showed that all parameters are non-parametric as $\mathrm{p}$ value is $\leq 0.05(\mathrm{p}=0.000)$.

Results: Total 99individuals were analyzed who had refractive errors. There were 60 female and 39male individuals. Avg age of individuals was 21 with standard deviation \pm 3 . Association of rang of accommodation between hypermetropes and emmetropes during point break, Rec point and Rang D the $p$ value is 0.001 in all points. Association of rang of accommodation between emmetropes and myopes during point break, Rec point and Rang $\mathrm{D}$ the $\mathrm{p}$ value is $0.142,0.224$ and 0.315 accordingly.

Conclusion: Association of rang of accommodation is significant between emmetropes and hypermetropes $(\mathrm{p}=0.001)$. But there is no significant association of rang of accommodation between Myopes and emmetropes $(\mathrm{p}=0.224)$.

Keywords: refractive errors, accommodation, presbyopia, crystalline lens, ciliary muscles
Volume 5 Issue 3 - 2021

\author{
Rashid Asghar Khan,' Adnan Afsar, ${ }^{2}$ \\ Muhammad Imshad Khan ${ }^{2}$ \\ 'Lecture Optometry, The University of Lahore Main campus \\ ${ }^{2} \mathrm{M}$ Phil Scholar Pakistan Institute of Community Ophthalmology, \\ Peshawar, Pakistan
}

\section{Correspondence: Adnan Afsar, M. Phil Scholar Pakistan Institute of Community Ophthalmology, Peshawar, Pakistan, Tel+92-334-9092917, Email adnanafsar55@gmail.com}

Received: January 29, 202 I | Published: July 02, 202 |
Abbreviations: VA, visual acuity; RE, refractive errors; COAVS, college of ophthalmology and allied vision Sciences; CL, contact Lens; DS, diopters Sphere (unit of power of sphere); DC, diopters cylinder (unit of power of cylinder); $1^{\circ}$, degree (unit of cylindrical axis angle); Pre-op, before procedure; Post-op, after procedure

\section{Introduction}

Worldwide, more than 150 million people are estimated to be visually impaired because of uncorrected refractive error, of which 8 million are functionally blind. Refractive error affected 1.45 billion people, or $27 \%$ of the world's population, in 2010. By the year 2020, it is estimated that 2.5 billion people, or one third of the world's population, will be affected by myopia alone. ${ }^{1}$ The refractive error profile of the United States adult population is less than 5\% hyperopic, about $30-40 \%$ myopic, and slightly more than half are emmetropic., ${ }^{2,3}$ Accommodation is the mechanism used by the pre-presbyopic eye to produce clear images on the retina of objects at different distances. This reflective mechanisms initiated by signals that activate the ciliary muscle, changing the shape of the crystalline lens and thus the power of the eye. ${ }^{4,5}$ These signals should include the needed focus direction whether positive or negative. ${ }^{6,7}$ The human eye has a maximum capacity of accommodation, known as accommodative amplitude (AA), which depends mainly on age. Ametropia is a potential factor influencing AA but studies have produced conflicting results. Some studies have reported greater AA in myopes than in emmetropes and hyperopes, while others found the opposite or no differences. ${ }^{8-11}$ Although most studies show that AA is larger in myopic eyes, results are not fully comparable because of differences in methodologies between studies.

Accommodative amplitude is usually measured clinically when the patient is wearing habitual correcting lenses. The optical effect of the correcting lens when stimulus vergence varies must be considered to give the appropriate measure of the accommodation response. We will refer to this well-known effect of correcting lenses as the "external effect," which has been studied previously and is normally taken into account. ${ }^{12-17}$ Amplitude of accommodation has been found variously to be reduced in myopes, increased in myopes, and unaffected by the refractive error. Again, although a reduced accommodative response is found in myopes, there is some controversy about whether responses worsen as myopia progresses or whether there is no relationship between progression and accommodative response. Accommodative dynamics, assessed by facility of accommodation measurements are reduced for distance viewing in myopes, but not for near work. ${ }^{1,18,19}$ 


\section{Objectives}

\section{Primary objective}

To study the range of accommodation in myopes and hypermetropes in comparison to normal population in different age groups.

\section{Secondary objective}

To study whether the range of accommodation differs in myopes and hypermetropes than normal population.

\section{Material and methods}

\section{Study design}

Comparative Cross-sectional study.

\section{Duration}

Study was conducted in six months after approval of synopsis.

\section{Study setting}

The institute for the study was college of ophthalmology and allied vision sciences mayo hospital Lahore.

\section{Sampling technique}

Non-probability, convenient sampling.

\section{Sample size}

Sample size of 99 patients 33 patients in each group is estimated by using $1 \%$ level of significance, $90 \%$ power of test, with expected $\%$ age of emmetropes as $73 \%$ and refractive error as $27 \%$.

$$
n=\frac{\left\{z_{1-\frac{\alpha}{2}} \sqrt{2 P(1-P)}+z_{1-\beta} \sqrt{P_{1}\left(1-P_{1}\right)+P_{2}\left(1-P_{2}\right)}\right\}^{2}}{\left(P_{1}-P_{2}\right)^{2}}
$$

$z_{1-\frac{\alpha}{2}}=$ confidence level $99 \%$

$Z_{1}-\beta=$ power of test $90 \%$

$\mathrm{P} 1=$ population proportion $\mathrm{I}=73 \%$

$\mathrm{P} 2=$ population proportion $\mathrm{II}=27 \%$

\section{Inclusion criteria}

Age group 10 to 55 years

Fully corrected myopia and hypermetropia.

\section{Exclusion criteria}

Uncooperative patients.

Mentally Retarded patients

Patients not willing

\section{Independent variable}

Age.

Gender

\section{Dependent variable}

Range of accommodation.
Refractive errors

Visual acuity

\section{Results}

\section{Age wise distribution of participants}

Amongst total study participants ( $\mathrm{n}=99$ ) Male participants were $39 \%$, while female participants of the study were

\begin{tabular}{|c|c|c|}
\hline \multicolumn{3}{|c|}{ Gender wise distribution } \\
\hline Male & 39 & $39 \%$ \\
\hline Female & 60 & $60 \%$ \\
\hline
\end{tabular}

Frequency distribution of mean break point amongst emmetropes and hypermetropes

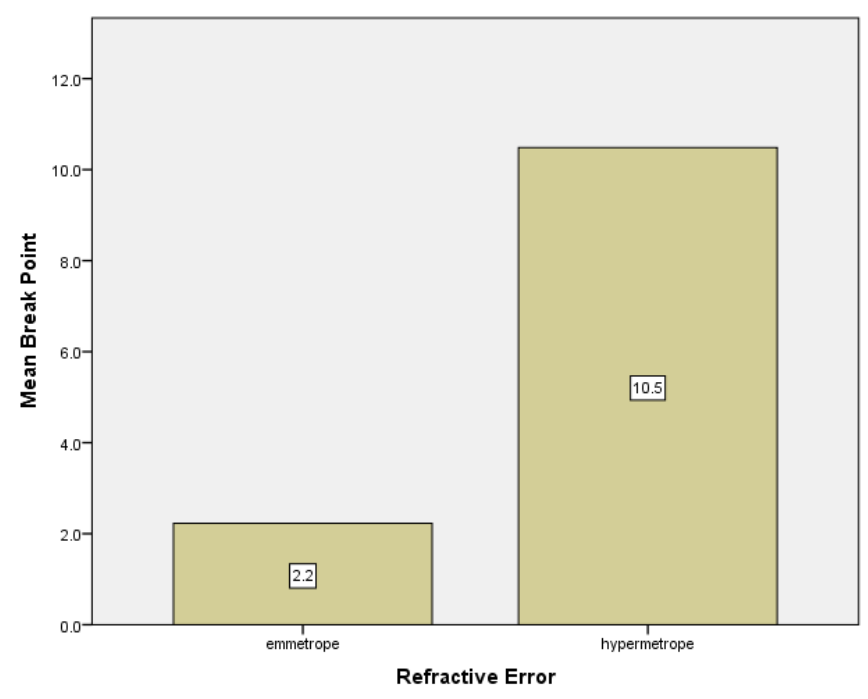

Association of rang of accommodation between hypermetropes and emmetropes during point break the $\mathrm{p}$ value is 0.001 .

\section{Graph showing comparison of mean recording point} amongst hypermetropes and emmetropes

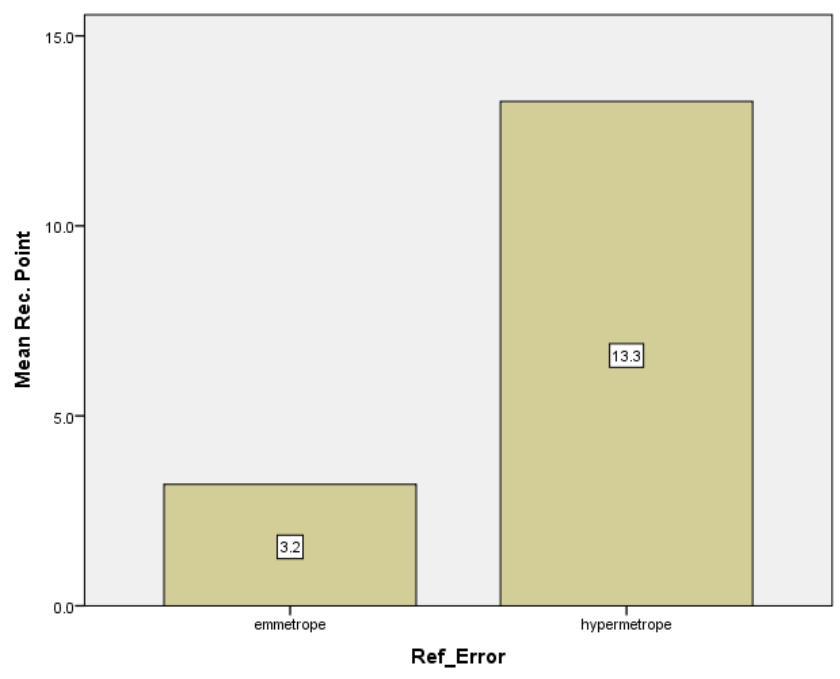

Association of rang of accommodation between hypermetropes and emmetropes during Rec point the $\mathrm{p}$ value is 0.001 . 
Mean range $D$ amongst emmetropes and hypermetropes

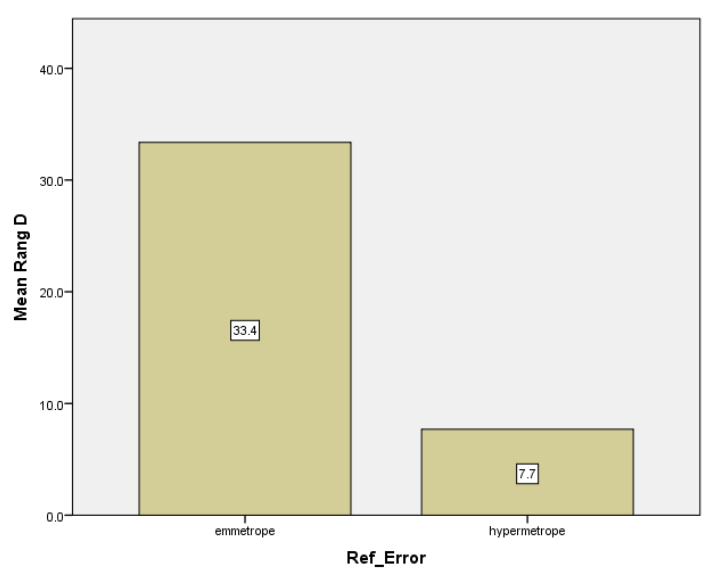

Total 99 individuals were analyzed who had refractive errors. There were 60 female and 39 male individuals. Avg age of individuals was 21 with standard deviation \pm 3 . Association of rang of accommodation between hypermetropes and emmetropes during point break, Rec point and Rang $\mathrm{D}$ the $\mathrm{p}$ value is 0.001 in all points.

\section{Frequency distribution of mean break point amongst emmetropes and myopes}

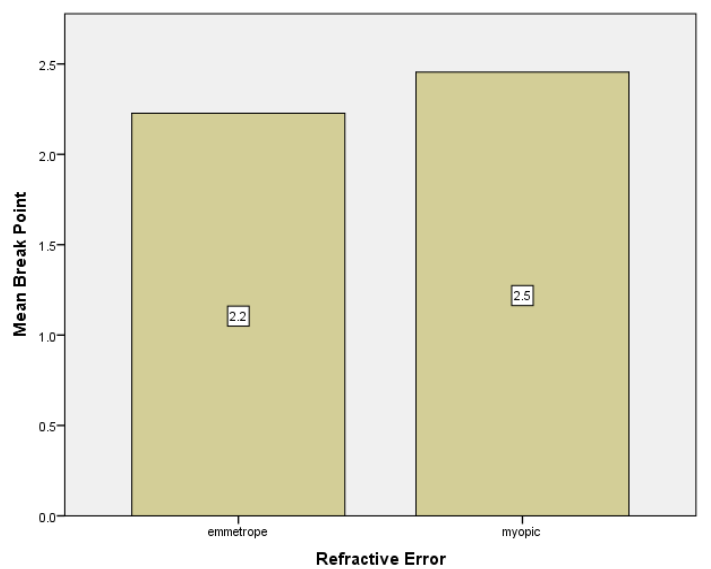

Association of rang of accommodation between emmetropes and myopes during point break, the $\mathrm{p}$ value is 0.142 .

\section{Graph showing comparison of mean recording point amongst myopes and emmetropes}

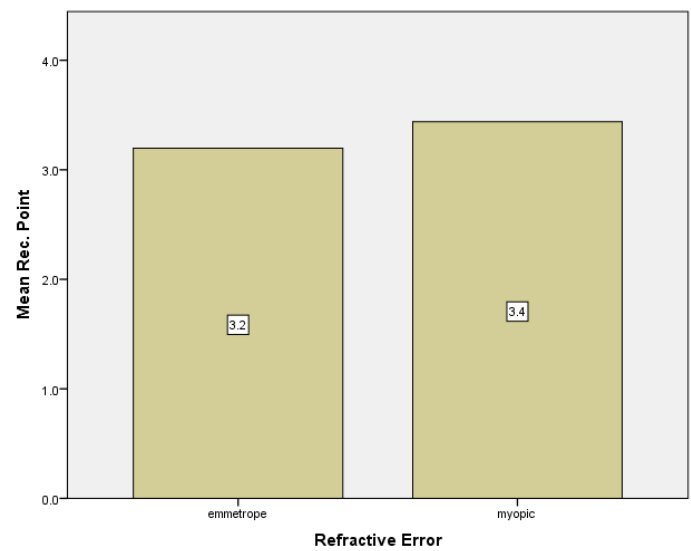

Association of rang of accommodation between emmetropes and myopes during Rec point the $\mathrm{p}$ value is 0.224 .

\section{Mean range $D$ amongst emmetropes and myopes}

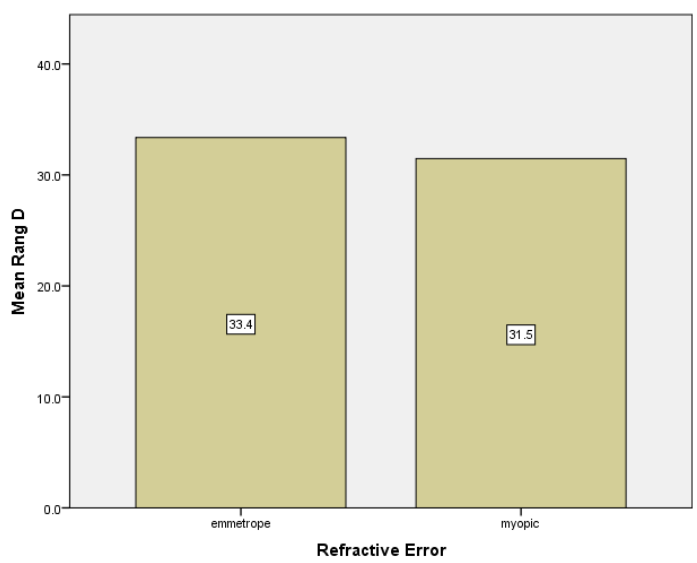

Association of rang of accommodation between emmetropes and myopes during Rang $\mathrm{D}$ the $\mathrm{p}$ value is 0.315 .

\section{Comparison of association of range of accommodation between myopes, hypermetropes and normal individuals}

Comparison of association of range of accommodation between myopes and normal individuals showed no significant difference as $p \geq 0.05$ and $p$ values were point break, rec point and rang $\mathrm{d}$ the $\mathrm{p}$ value is $0.142,0.224$ and 0.315 accordingly. There are many studies which showed similar results. In a study which was conducted by American Society of Optometry showed that there is difference between range of accommodation in all refractive errors (myopes, hypermetropes) and normal population. This study includes all type of spherocylindrical errors., While there is significant difference between hypermetropes and normal individuals as $\mathrm{p}$ value is 0.001 . Our results that there is significant difference between range of accommodation in all refractive errors (myopes, hypermetropes) and normal population are not new but to the best of our information this is only studies which showed the comparison between these two procedures. While there is significant difference between hypermetropes and normal individuals as $p$ value is $>0.05$.

\section{Discussion}

Worldwide, more than 150 million people are estimated to be visually impaired because of uncorrected refractive error, of which 8 million are functionally blind. Refractive error affected 1.45 billion people, or $27 \%$ of the world's population, in 2010 . By the year 2020 , it is estimated that 2.5 billion people, or one third of the world's population, will be affected by myopia alone. ${ }^{1,2}$ The refractive error profile of the United States adult population is less than 5\% hyperopic, about $30-40 \%$ myopic, and slightly more than half are emmetropic. This study describes the difference range of accommodation in all refractive errors (myopes, hypermetropes) and normal population. This study recommends to correctly check the rang of accommodation of everyone so that examiner could get better visual results. This study also led to investigate its clinical features and new clinical methodologies in relation of this topic of rang of accommodation. This study also showed that there is significant difference between range of accommodation in all refractive errors (myopes, hypermetropes) and normal population. Accommodation refers to the ability of young eyes to actively bring into focus objects that are at different distances. For non-presbyopic eyes with no disabilities, accommodation is fast and precise. This study has shown no significant difference (equal visual results) as $\mathrm{p}$ value is $>0.05$ in Myopes and Normal individuals. 
Accommodation is the mechanism used by the pre-presbyopic eye to produce clear images on the retina of objects at different distances. This reflexive mechanism ${ }^{1,2}$ is initiated by signals that activate the ciliary muscle, changing the shape of the crystalline lens and thus the power of the eye. These signals should include the needed focus direction whether positive or negative. ${ }^{4}$ The human eye has a maximum capacity of accommodation, known as accommodative amplitude (AA), which depends mainly on age. Ametropia is a potential factor influencing AA but studies have produced conflicting results. Some studies have reported greater AA in myopes than in emmetropes and hyperopes, while others found the opposite or no differences. ${ }^{5,6}$ Although most studies show that AA is larger in myopic eyes, results are not fully comparable because of differences in methodologies between studies. Different choices of reference plane for specifying vergence of the far and near points are of importance for our investigation of potential optical explanations for variability in results reported in the literature. Accommodative amplitude is usually measured clinically when the patient is wearing habitual correcting lenses. Myopia is the refractive anomaly of the eye in which the conjugate focus of the retina is at some finite point in front of the eye, when the eye is not accommodating. ${ }^{15}$ Instantaneous data for all 40 individuals in the Davis study exposed the following: Rec point of accommodation, $X=1.43$ D, S.D. $=1.03 \mathrm{D}$; far point, $\mathrm{X}=\mathrm{JX} 28 \mathrm{D}$, S.D. $=0.41 \mathrm{D}$; and Rang D, $X=10.21$ D, S.D. $=2.10$ D. Rec point and far point quantities were significantly correlated in Hypermetropes ( $r=+0.48, \mathrm{p}<0.01$ ), demonstrating that part of the patient's difference in the Rec point of accommodation is linked to subjects' differences in the far point of accommodation. Associations between the Rec point and Rang D point of accommodation $(r=+0.08)$ and between Rec point and point break of accommodation $(r=+0.07)$ were not significant.

\section{Conclusion}

Our study showed that there is significant difference between range of accommodation in all refractive errors (myopes, hypermetropes) and normal population. Association of rang of accommodation between hypermetropes and emmetropes during point break, Rec point and Rang $\mathrm{D}$ the $\mathrm{p}$ value is 0.001 in all points. Association of rang of accommodation between emmetropes and myopes during point break, Rec point and Rang D the $\mathrm{p}$ value is $0.142,0.224$ and 0.315 accordingly. Association of rang of accommodation is significant between emmetropes and hypermetropes $(p=0.001)$. But there is no significant association of rang of accommodation between Myopes and emmetropes $(p=0.224)$. Our study suggests that there is difference between range of accommodation in all refractive errors (myopes, hypermetropes) and normal population. This study recommends to correctly check the rang of accommodation of everyone so that examiner could get better visual results. This study will also lead to investigate its clinical features and new clinical methodologies in relation of this topic of rang of accommodation.

\section{Acknowledgments}

The entire period for thesis writing was tedious and it is certainly not done without support and contribution of many people. First of all, thanks to Allah for providing me strength to complete this thesis work. I would like to express my gratitude to my family, especially father, mother and brothers for their moral support in order to complete this study. Without their support and understanding, it would not have been possible for me to get my educational goals.

\section{Conflicts of interest}

We declare no financial interest or any conflict of interest.

\section{References}

1. Woodman EC, Read SA, Collins MJ. Axial length and choroidal thickness changes accompanying prolonged accommodation in myopes and emmetropes. Vision research. 2012;72:34-41.

2. Woodman-Pieterse EC, Read SA, Collins MJ, et al. Anterior scleral thickness changes with accommodation in myopes and emmetropes. Experimental eye research. 2018;177:96-103.

3. Maiello G, Kerber KL, Thorn F, et al. Vergence driven accommodation with simulated disparity in myopia and emmetropia. Experimental eye research. 2018;166:96-105.

4. Schilling T, Ohlendorf A, Varnas SR, et al. Peripheral Design of Progressive Addition Lenses and the Lag of Accommodation in Myopes. Investigative ophthalmology \& visual science. 2017;58(9):3319-3324.

5. Neroev VV, Tarutta EP, Arutyunyan SG, et al. Wavefront aberrations and accommodation in myopes and hyperopes. Vestnik oftalmologii. 2017;133(2):5-9.

6. Gualdi L, Gualdi F, Rusciano D, et al. Ciliary Muscle Electrostimulation to Restore Accommodation in Patients With Early Presbyopia: Preliminary Results. Journal of refractive surgery. 2017;33(9):578-583.

7. Aldossari H, Suheimat M, Atchison DA, et al. Effect of Accommodation on Peripheral Eye Lengths of Emmetropes and Myopes. Optometry and vision science: official publication of the American Academy of Optometry. 2017;94(3):361-369.

8. $\mathrm{Xu} \mathrm{J}$, Zheng Z, Drobe B, et al. The effects of spatial frequency on the accommodation responses of myopes and emmetropes under various detection demands. Vision research. 2015;115(Pt A):1-7.

9. Schmid KL, Strang NC. Differences in the accommodation stimulus response curves of adult myopes and emmetropes: a summary and update. Ophthalmic \& physiological optics: the journal of the British College of Ophthalmic Opticians. 2015;35(6):613-21.

10. Liu Y, Lv H, Jiang $\mathrm{X}$, et al. Intraocular Pressure Changes during Accommodation in Progressing Myopes, Stable Myopes and Emmetropes. PloS one. 2015;10(10):e0141839.

11. Goldberg DB. Computer-animated model of accommodation and presbyopia. Journal of cataract and refractive surgery. 2015;41(2):437-445.

12. Faramarzi A, Bagheri A, Karimian F, et al. Correlation between ocular biometry and amplitude of accommodation in early presbyopia. European journal of ophthalmology. 2015;25(4):298-301.

13. Yan L, Huibin L, Xuemin L. Accommodation-induced intraocular pressure changes in progressing myopes and emmetropes. Eye. 2014;28(11):1334-1340

14. Thompson-Hollands J, Kerns CE, Pincus DB, et al. Parental accommodation of child anxiety and related symptoms: range, impact, and correlates. Journal of anxiety disorders. 2014;28(8):765-773.

15. Reilly MA. A quantitative geometric mechanics lens model: insights into the mechanisms of accommodation and presbyopia. Vision research. 2014;103:20-31.

16. Kirkwood BJ, Kirkwood RA. Accommodation and presbyopia. Insight 2013;38(3):5-8.

17. Croft MA, McDonald JP, Katz A, et al. Extralenticular and lenticular aspects of accommodation and presbyopia in human versus monkey eyes. Investigative ophthalmology \& visual science. 2013;54(7):5035-5048. 
18. Van de Sompel D, Kunkel GJ, Hersh PS, et al. Model of accommodation contributions of lens geometry and mechanical properties to the development of presbyopia. Journal of cataract and refractive surgery. 2010;36(11):1960-1971.
19. Read SA, Collins MJ, Woodman EC, et al. Axial length changes during accommodation in myopes and emmetropes. Optometry and vision science: official publication of the American Academy of Optometry. 2010;87(9):656-662. 